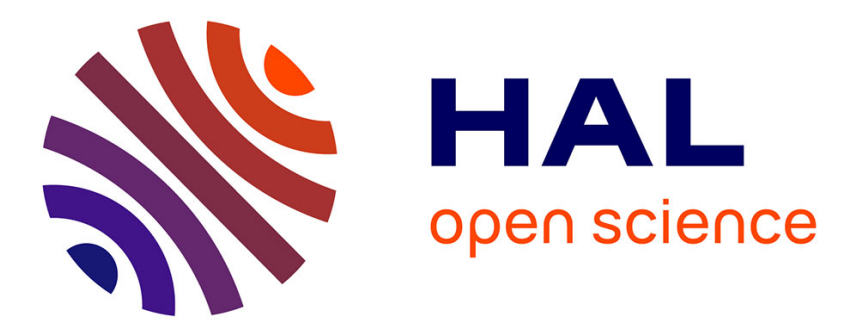

\title{
Calculation of Net Emission Coefficient for High Intensity Discharge Lamps
}

Georges Zissis, Rawan Al Youssif, Antoine Sahab, Walid Malaeb, Mohamad Hamady

\section{- To cite this version:}

Georges Zissis, Rawan Al Youssif, Antoine Sahab, Walid Malaeb, Mohamad Hamady. Calculation of Net Emission Coefficient for High Intensity Discharge Lamps. Light \& Engineering, 2020, 28 (3), pp.80-86. hal-02867062

\section{HAL Id: hal-02867062 https://hal.science/hal-02867062}

Submitted on 7 Jul 2020

HAL is a multi-disciplinary open access archive for the deposit and dissemination of scientific research documents, whether they are published or not. The documents may come from teaching and research institutions in France or abroad, or from public or private research centers.
L'archive ouverte pluridisciplinaire HAL, est destinée au dépôt et à la diffusion de documents scientifiques de niveau recherche, publiés ou non, émanant des établissements d'enseignement et de recherche français ou étrangers, des laboratoires publics ou privés. 


\title{
CALCULATION OF NET EMISSION COEFFICIENT FOR HIGH INTENSITY DISCHARGE LAMPS
}

\author{
Rawan Al Youssif ${ }^{1}$, Antoine Sahab ${ }^{2}$, Georges Zissis², Walid Malaeb ${ }^{1}$, \\ and Mohamad Hamady1,3 \\ ${ }^{1}$ Department of Physics, Faculty of Science, Beirut Arab University, Lebanon \\ ${ }^{2}$ University of Toulouse; UPS, INPT, CNRS; LAPLACE \\ (LaboratoirePlasmas et Conversion d'Energie), France \\ ${ }^{3}$ Faculty of Sciences, Lebanese University, El-Hadath, Beirut, Lebanon \\ E-mail: hamadymohamad@gmail.com
}

\begin{abstract}
There is still great interest in studying high intensity discharge (HID) lamps despite the great development of other light sources like light emitting diodes (LEDs). Basic equations and numerical formulations allow calculating important terms such as the net emission coefficient (NEC) that plays an important role in understanding the radiation behaviour of these lamps. These lamps are considered to be at high pressure and the produced plasma was found to be at local thermodynamic equilibrium (LTE). The volume of the lamp is meshed into small cells and the total number of cells represents a compromise between correct results and calculation time. Each cell has its own local absorption and emission coefficient that applies to its position in the discharge. Line profile is calculated by two profiles convolution: one is Lorentz's and the second one is a quasi-static profile. Ray tracing technique is used to resolve the radiation transport for the visible and ultra violet (UV) spectrum. The NEC is thus calculated and compared with other models for a pure mercury discharge. In addition, additional photometric properties of the lamp are obtained.

Keywords: high intensity discharge (HID), radiation transport, ray tracing, net emission coefficient (NEC), line broadening, local thermodynamic equilibrium (LTE), intensity, flux, luminous flux, luminous efficacy, correlated colour temperature (CCT)
\end{abstract}

\section{INTRODUCTION}

High intensity discharge (HID) lamps are compact gas discharges $((1-100) \mathrm{mm}$ or more in length) that operate at pressures of order $1 \mathrm{~atm}$ or greater. These lamps are of great interest due to their different applications like projectors and television projection systems [1]. These lamps are excellent sources of different radiations and over time cannot be replaced by another type of light sources, like light emitting diodes (LEDs), for example.

Radiation is one of the three mechanisms of energy transfer and it can be considered as the product of several emission and absorption processes in a high pressure discharge in relation with the continuous and discontinuous spectrum. The characteristics of this spectrum depend on the state of the electrical discharge like the size, temperature, pressure, or composition. The knowledge of radiative properties is a key factor in achieving quantification of the energy exchanges. In simulation these radiative exchanges are often quantified by the divergence of the radiative flux, which characterizes the energy transfer by radiation everywhere in the plasma, and which can be easily estimated using the "Net Emission Coefficient (NEC)", a quantity that can also be used in the prediction of energy balance [2].

Computer modelling is an accurate and inexpensive way to determine the NEC in high-pressure gas discharge lamps. However, it is not possible to find 
an exact analytical solution to highly nonlinear integer-differential radiative transfer equation [3]. During several decades, a well-known phenomenon was working on generating radiation-using formalism to describe them [4].

Leibermann and Lowke calculated the NEC using these methods and the results obtained from the calculations, and Sevastyanenko determined the partial characteristics [5,6]. Moreover, Lowke and Capriotti determined the diffusion approximation [7].

Other methods calculated the NEC using semi-empirical formula $[8,9]$. Also BouAoun et $a l$. calculated the NEC using the discrete ordinate method, where this method has been applied to numerous radiative problems with significant accuracy, and it is an analogous to the finite volume discretization in directional space. In this paper, we have used another method to calculate this index based on ray tracing and, to our knowledge, there is no direct calculation on the NEC has been done using this technique [10].

For this purpose, we have focused in our work on the calculation of the power radiated by these lamps using ray tracing technique for pure mercury $\mathrm{Hg}$ as a vehicle of study given that $\mathrm{Hg}$ has well known characteristics.

In these calculations, we have considered that the discharge has a cylindrical symmetry and we assumed that the plasma is at local thermodynamic equilibrium (LTE). The only knowledge of the temperature profile and pressure is sufficient to calculate the chemical composition (local emission and absorption coefficient) of plasma and to the mechanisms to increase of spectral lines. In this technique, the discharge is divided into elementary cells responsible for launching rays in all directions, and the radiative transfer equation (RTE) is resolved for each ray. If there is no absorption exists in each mesh of the discharge then the NEC equals to local emission coefficient $\varepsilon$. However, as absorption exists, the NEC and $\varepsilon$ have different values, which show the importance of this study. Therefore, the net emission coefficient and the photometric properties of the lamp are deduced.

\section{RADIATION TRANSPORT}

As radiation is transported in the plasma, it contributes to the energy balance of the discharge. The equation of radiative transfer describes the change of spectral intensity $I_{\lambda}\left[\mathrm{W} \cdot \mathrm{m}^{-3} \cdot \mathrm{sr}^{-1}\right]$ along a path $x$. When the medium emits and absorbs radiation without scattering this equation is written:

$$
\frac{d I_{\lambda}}{d x}=\varepsilon_{\lambda}(\lambda, x)-\kappa(\lambda, x) I_{\lambda},
$$

where $\varepsilon_{\lambda}(\lambda, x)$ is the local spectral emissivity [W' $\left.\mathrm{m}^{-4} \mathrm{sr}^{-1}\right]$ and $\kappa\left[\mathrm{m}^{-1}\right]$ is the local spectral absorption index. For a spectral line corresponding to a transition between atomic levels $u$ and $l, \varepsilon_{\lambda}$ is firstly calculated from plasma composition by using the following equation:

$$
\varepsilon(\lambda)=\frac{\mathrm{hc}}{4 \pi} \frac{A_{\mathrm{ul}} \mathrm{n}_{u} P(\lambda)}{\lambda_{0}},
$$

where $P(\lambda)$ is the normalized spectral line profile.

In HID lamps, as the pressure is relatively high, the LTE assumption is considered valid. Therefore, $\kappa$ is calculated by using $B_{\lambda}$ (the Planck function) by:

$$
\kappa(\lambda, T)=\frac{\varepsilon_{\lambda}(\lambda, T)}{B_{\lambda}(T)},
$$

where

$$
B_{\lambda}(T)=\frac{2 h c^{2}}{\lambda^{5}}\left[\exp \left(\frac{\mathrm{hc}}{\lambda \mathrm{kT}}\right)-1\right]^{-1} .
$$

Without knowing the plasma composition, the resolution of the radiative transfer equation is impossible. In the case of a HID lamp, this composition is calculated assuming that local thermodynamic equilibrium (LTE) exists, and that pressure is uniform in the discharge. Pressure and local temperature are determined from spectroscopic measurements. Following equation corresponds to the temperature profile across the discharge:

$$
T(r)=T_{c}-\left(T_{c}-T_{w}\right)\left(\frac{r}{R}\right)^{\beta},
$$

where $T_{c}$ is the central temperature, $T_{w}$ is the wall temperature, $\beta$ is the parameter that describes the radial distribution of the discharge, and $R$ is the discharge radius.

Applying the equations of state for ideal gas (Boltzmann and Saha laws), we are able to calculate the plasma composition of the discharge. On the other hand, for the spectral line profile only pressure broadening is taken into account (Doppler broadening is considered negligible). Pressure broadening is due to the collisions of emitting atoms with other atoms (neutral Van der Waals or resonance broadening) or charged particles (Stark broadening). In the 
impact approximation, pressure broadening is described by a Lorentz's profile:

$$
P(\lambda)=\frac{\Delta \lambda_{1 / 2}}{\pi} \frac{1}{\left(\lambda-\lambda_{o}-\Delta\right)^{2}+\left(\Delta \lambda_{1 / 2}\right)^{2}},
$$

where

$$
\begin{gathered}
\Delta \lambda_{1 / 2 R}=C_{R} N \Delta \lambda_{1 / 2 s}=C_{S} N_{e}, \Delta_{S}=0.87 \Delta \lambda_{1 / 2} s, \\
\Delta \lambda_{1 / 2 W}=C_{W} N, \Delta_{W}=0.36 \Delta \lambda_{1 / 2 W} .
\end{gathered}
$$

The resulting total line profile is obtained by the convolution of the Lorentz (6) and quasi-static (7) profiles. Atomic and broadening constants for the mercury spectral lines are taken from Stromberg [8].

$$
P_{Q S}(\lambda)=\left\{\begin{array}{c}
\frac{1}{2} \sqrt{\frac{\Delta \lambda_{Q S}}{\left(\lambda-\lambda_{0}\right)^{3}}} \cdot \\
\cdot \exp \left(-\frac{\pi}{4} \frac{\Delta \lambda_{Q S}}{\lambda-\lambda_{0}}\right) \text { if } \lambda \succ \lambda_{0}, \\
0 \text { if } \lambda \leq \lambda_{0}
\end{array}\right.
$$

where

$$
\Delta \lambda_{Q S}=C_{Q S} * N^{2} .
$$

To resolve the radiation transport that takes place inside the lamp, it volume is meshed into small cuboid elements. A set of the $N$ isotropic rays is launched in each element. Each ray will cross each volume element until it escapes from the mesh. The index of each traversed element volume travelled by rays, and the distance within each of them are calculated and stored.

Each ray contributes to the output radiation flux. The radiant flux that emerges from the emitting element volume of each ray is calculated by:

$$
\Phi_{\text {out }}=I \cdot \Delta A \cdot \cos \theta \cdot \Delta \Omega,
$$

where $\Delta A$ is the surface crossed by the ray, $\Delta \Omega$ is the solid angle, $\theta$ is the angle between the ray and the normal to the surface, and $I$ is the output intensity of the emitting element volume (Fig. 1).

In a mesh, the output intensity $I$ is derived from the integral solution of radiative transfer equation (9). Taking into account that each finite volume " $i$ " is considered as homogeneous, the radiant intensi-

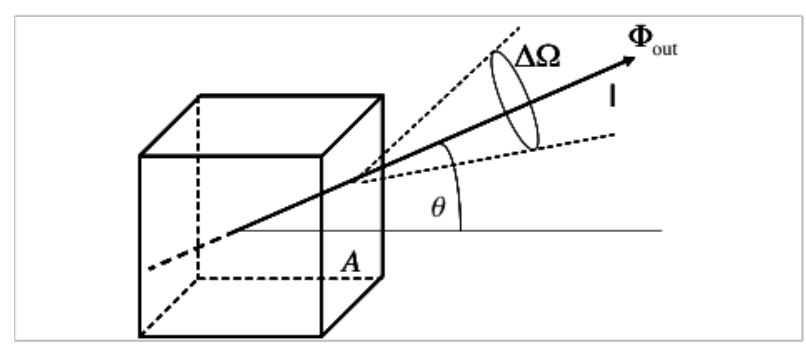

Fig. 1. Output radiant flux of the emitting cell

ty $I$ in the direction of the ray at a boundary point $P$ takes the following form:

$$
\begin{gathered}
I_{\lambda}\left(\lambda, x_{i+1}\right)=I_{\lambda}\left(\lambda, x_{i}\right) \exp \left(-\kappa(\lambda, i) \Delta x_{i}+\right. \\
+\frac{\varepsilon_{\lambda}(\lambda, i)}{\kappa(\lambda, i)}\left(1-\exp \left(-\kappa(\lambda, i) \Delta x_{i}\right)\right),
\end{gathered}
$$

where $\Delta x_{i}$ is the length crossed in each finite volume " $i$ ".

The ray will cross all cells in the mesh along its path. The output radiant flux $\Phi_{\text {out }}(8)$ of the emitting mesh represents the input radiant flux $\Phi_{i n}$ for the next crossed mesh. This flux will be absorbed before leaving the mesh. Therefore, the radiant flux $\Phi_{\text {out }}$ that goes out from the next crossed mesh is:

$$
\Phi_{\text {out }}=\Phi_{\text {in }} \cdot \exp (-\kappa \cdot \Delta x),
$$

where $\Delta x$ is the distance crossed and $\kappa$ is the absorption index of the crossed mesh.

Therefore, the absorbed radiant flux $\Phi_{\text {absorbed }}$ in the mesh crossed is calculated by:

$$
\Phi_{\text {absorbed }}=\Phi_{\text {in }}-\Phi_{\text {out }} .
$$

The quantity of $\Phi_{\text {out }}$ in (10) represents $\Phi_{\text {in }}$ for the next crossed mesh. Therefore, we continue calculating $\Phi_{\text {out }}$ as in (10) and $\Phi_{\text {absorbed }}$ as in (11) in every mesh crossed until the ray leaves the lamp. At this

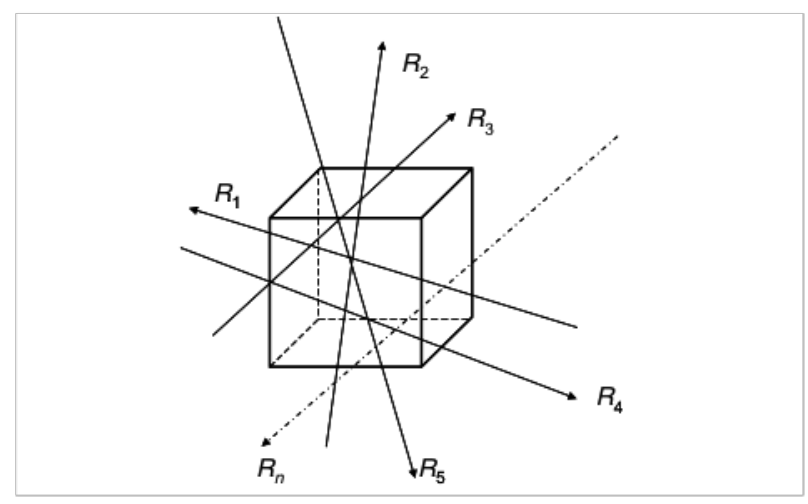

Fig. 2. Rays depose energy in a cell 

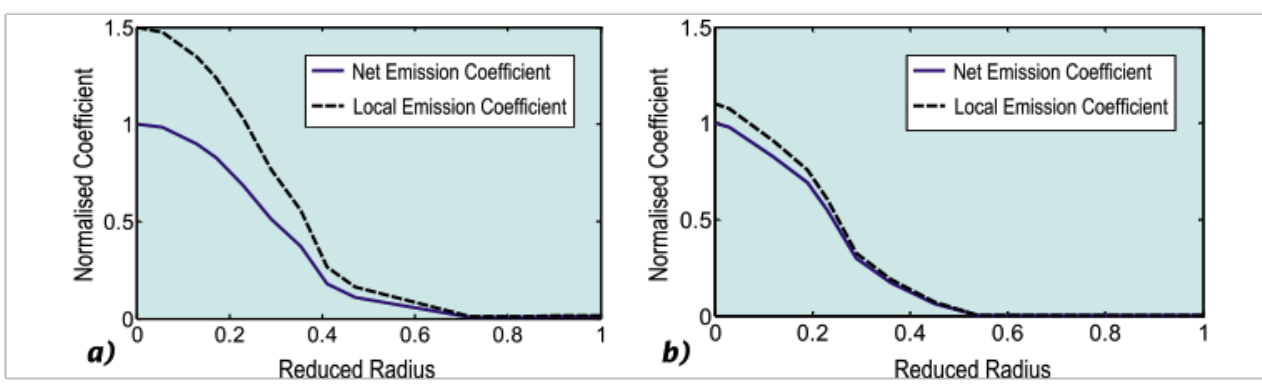

Fig. 3. Local emissivity and net emission coefficient (NEC) comparison at $435 \mathrm{~nm}$ (a) and $577 \mathrm{~nm}$ (b) wavelengths
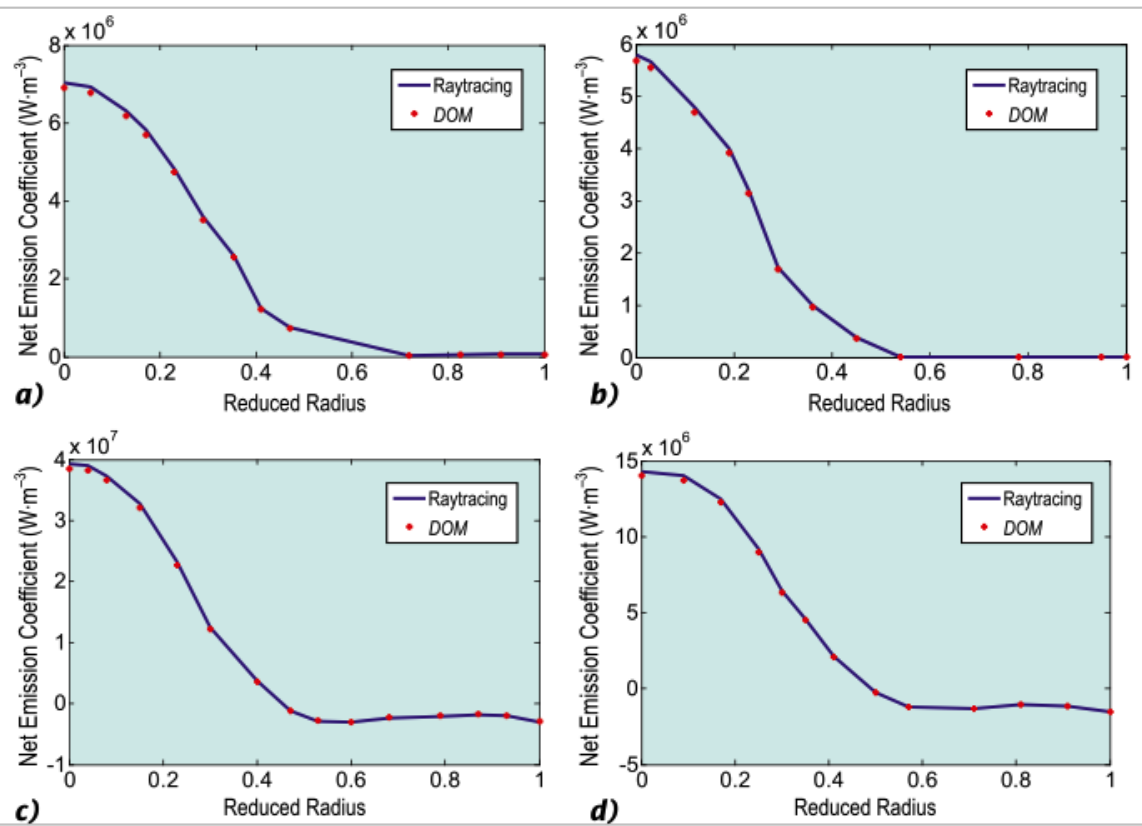

moment, $\Phi_{\text {out }}$ is stored and it represents the contribution of the ray in the radiant flux calculation. Another ray is selected and the calculation is repeated until all rays are launched from all cells. As a result, different rays cross whole mesh and each ray deposes certain radiant flux $\Phi_{\text {absorbed }}$ in the mesh (Fig. 2).

So, the net emission coefficient is calculated by:

$$
\varepsilon_{N}=\varepsilon-\frac{\sum \Phi_{\text {absorbed }}}{4 \pi \Delta V},
$$

where $\varepsilon$ is the local emissivity of the mesh and $\Delta V$ is the mesh volume.

The total radiant flux $\Phi_{T}$ escaping from the lamp is:

$$
\Phi_{T}=\sum_{n=1}^{N} \sum_{k=1}^{n b_{-}} \sum_{j=1}^{n b_{y}} \sum_{i=1}^{n b_{x}} \Phi_{\text {out }},
$$

where $n b_{x}, n b_{y}, n b_{z}$ are the number of cells along $x$-axis, $y$-axis and z-axis respectively, and $N$ is the total number of rays emitted from each cell.

So, the luminous flux is:

$$
\Phi_{v i s}=K_{m} \int_{\lambda} V(\lambda) \Phi_{\lambda}(\lambda) d \lambda,
$$

where $V(\lambda)$ is the spectral luminous efficiency of the average eye for photopic vision and $K_{m}=683 \mathrm{~lm} / \mathrm{W}$. Then the luminous efficacy is:

$$
\eta=\frac{\Phi_{\text {vis }}}{P_{\text {electric }}},
$$

where $P_{\text {electric }}$ represents the electric power applied to the lamp. Based on the radiant flux of each line and using the colour-matching functions we can calculate CCT and chromaticity coordinates.

\section{RESULTS AND DISCUSSION}

In this work, pure mercury HID lamp has been studied. The lamp is represented by a cylindrical discharge where cylinder has been divided (or meshed) into small cells. We have divided our work into three parts. In the first part, we have chosen to mesh the cylinder into 35 cells on the radial direction surface and 13 cells along the axial direction. While Galvez [12] has launched 100 rays from each cell, we have previously found that launching 62 rays are enough [13]. This represents a good 


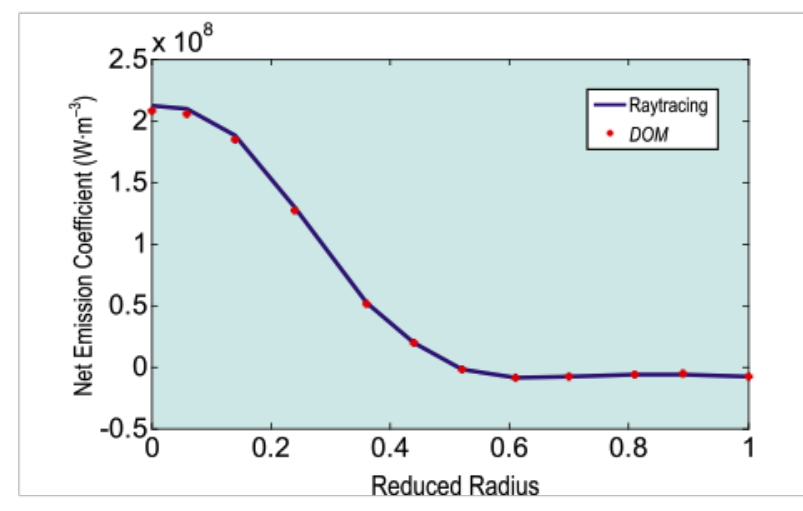

Fig. 5. Comparison of total NEC using ray tracing and DOM method for all spectral lines

compromise between Galvez calculation and time consumption. At the end of this part, the crossed distance in the emitting and crossed cells, the solid angles as well as the areas crossed with the corresponding angles are saved in a separate file.

In the second part, we assigned for each cell its temperature. Therefore, cells on the axial direction will have same temperature while the temperature of cells on the radial direction will be a parabolic distribution as in equation (5) according to their position. Once cell's temperature is known, local absorption index and emissivity are calculated.

In the third part, we resolve the radiation transport equation and start to get results by using equations (8-15). This way of calculation provides the model by ability to produce results more quickly compared to other methods. For example, if chemical composition changes, then we change part two without doing any change in part one while in other methods you should start again from scratch all your calculations.

In our calculations, we will study a discharge known as SC (standard conditions) discharge. This discharge is typical for a high-pressure mercury lamp used as a light source. In Table 1, we summarize the parameters of the SC discharge.

The NEC represents the difference between the emitted and the absorbed energy in each cell. Therefore, it is important to compare in each cell the NEC with the energy due to the local emissivity to make sure that absorption is taking place in each cell and the method is working reasonably which means comparing the value of equation (2) with the results of equation (12). Fig. 3 shows the results for the spectral line $435 \mathrm{~nm}$ and $577 \mathrm{~nm}$. Although the spectral line are not absorbed in a constant ratio, but the influence of absorption can be easily deduced
Table 1. Parameters of the Studied Discharge

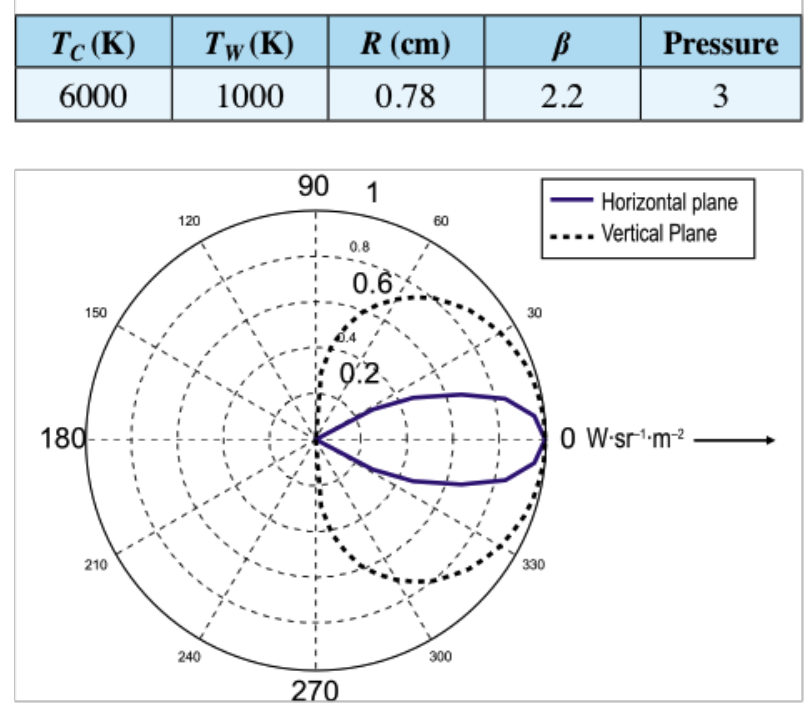

Fig. 6. Normalised distribution intensity curves on the horizontal and vertical surfaces

for these spectral lines. Same behaviour was seen for other spectral lines.

Bouaoun et al. [10] have also calculated the total NEC using DOM method by summing the NEC of each spectral line that exists in the discharge. His results were compared with Stomberg's formula. Both results were comparable and it is sufficient in this work to compare with one of these models. The NEC for each spectral line was calculated for the same SC discharge (Table 1) and compared with the results of Bouaoun et al. [10] who used the same discharge. The good agreement between both results is clear, as it is shown in Fig. 4.

The total NEC index is calculated for all spectral lines that exist in the discharge, which are tabulated in Stromberg [8]. The good agreement for the total NEC is seen with Bouaoun et al.[10] and it is shown in Fig. 5.

Our main objective in this research is to calculate the NEC and compare it with other models in order to provide a new tool to calculate this term in future applications. However, the technique used produces not only the NEC, but also the spectral results, which are the bases of calculation of the photometric properties of any lamp as it is shown in $[11,13]$. For example, for the studied SC discharge, the radiant flux is first calculated from each spectral line in the discharge using (13). Then, the luminous flux of each spectral line is obtained by using (14). The luminous efficacy calculated from equation (15) was equal $80 \mathrm{~lm} / \mathrm{W}$. This efficacy is comparable with the experimental results of a pure Hg lamp. 
The CCT for the discharge was received to be 4355 $\mathrm{K}$. This CCT is in accordance to the impression of white-blue colour known for pure Hg HID lamps. The photometric curve on the horizontal and vertical surface of the lamp is shown in Fig. 6.

\section{REFERENCES}

1. Derra G., Moench H., Fischer E., Giese H., Hechtfischer U., Heusler G., Koerber A., Niemann U., Noertemann F.-C., Pekarski P., Pollmann-Retsch J., Ritz A., Weichmann U. UHP lamp systems for projection applications // Journal of Physics D: Applied Physics, 2005. V38, \#3, pp. 2995.

2. Cressault Y., Teulet P., Zissis G. Radiative properties of ceramic metal-halide high intensity discharge lamps containing additives in argon plasma / / Japanese Journal of Applied Physics, 2016. V55, \#7S2, pp. 07LB05.

3. Simonet F., Aubes M., Elloumi H., Sarroukh H. Optimization of the spectruml flux computation for cylindrical discharges // Journal of Quantitative Spectroscopy and Radiative Transfer, 1999. V61,\#2, pp. 197.

4. Lochte-Holtegreven W. Plasma Diagnostics. North Holland Publishing Company, 1968.

5. Leibermann R.-W., Lowke, J.-J. Radiation emission coefficients for sulfur hexafluoride are plasmas // Journal of Quantitative Spectroscopy and Radiative Transfer, 1976. V16, \#3, pp. 253.

6. Sevast'yanenko V.-G., Soloukhin R.-I., Golovnev I.-F., Zamurayev V.-P., Katsnel'son V.-P., Koval'skaya,
G.-A., Goulard R. Radiative Heat Transfer in High Temperature Gases. Springer, 1987.

7. Lowke J.-J., Capriotti E.-R. Calculation of temperature profiles of high-pressure electric arc using the diffusion approximation for radiation transfer // Journal of Quantitative Spectroscopy and Radiative Transfer, 1969. V9, \#2, pp. 207.

8. Stromberg H.-P., Schafer R. Time-dependent behaviour of high-pressure mercury discharges // Journal of Applied Physics, 1983. V54, \#8, pp. 4338.

9. Jones B.-F., Mottram D.-A.-J. A semi-empirical formula to describe the net emission coefficient of self-absorbed spectruml lines for use in modeling high-pressure discharge lamps // Journal of Physics D: Applied Physics, 1981. V14,\#7, pp. 1183.

10. Bouaoun M., Elloumi H., Charrada K., Rhouma B.-E.-H., Stambouli M. Discrete ordinates method in the analysisof the radiative transfer in high intensity discharge lamps // Journal of Physics D: Applied Physics, 2005. V38, \#22, pp. 4053.

11. Hamady M., Lister G.-G., Zissis G. Calculations of visible radiation in electrodeless HID.

12. Lamps // Journal of Lighting Research and Technology, 2016. V48, \#4, pp. 502.

13. Galvez M. Ray tracing model for radiation transport in three-dimensional LTE systems // Journal of Physics D: Applied Physics, 2005. V38,\#17, pp. 3011.

14. Hamady M., Lister G.-G., Aubès M., Zissis G. Study of photometric properties of high-pressure mercury discharge wit thallium iodide additives (HgT $l \mathrm{I})$ using the ray-tracing method // Journal of Physics D: Applied Physics, 2011. V44, \#10, pp. 5201.

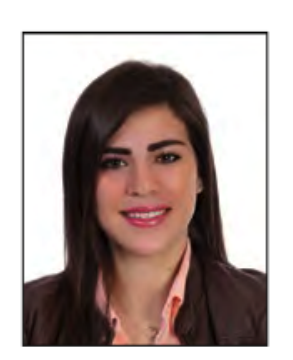

\section{Rawan Al Youssif}

received her bachelor degree in Physics from Beirut Arab University in June 2015, working currently to finish her Master Degree in Physics. Rawan's research interest includes the high intensity discharged lamps (HID), and how to calculate Net Emission Coefficient (NEC) using ray-tracing technique. She is a physics teacher at Saida High School - Abra with a five-year experience

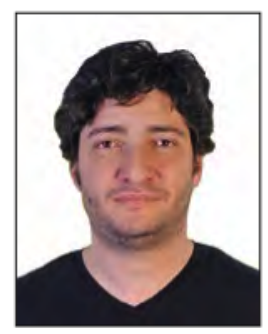

\section{Antoine Sahab}

joined "Light \& Matter" research group at LAPLACE laboratory as Ph.D. student at Paul Sabatier University, Toulouse, France where he received as well his Master's Degree. His research work is based on studying plasmas discharges, mainly plasma parameters and radiations. In addition to his scientific background, Mr. Sahab enjoys business skills where he is currently accounting and operation manager at Solid Worx SARL, Lebanon 


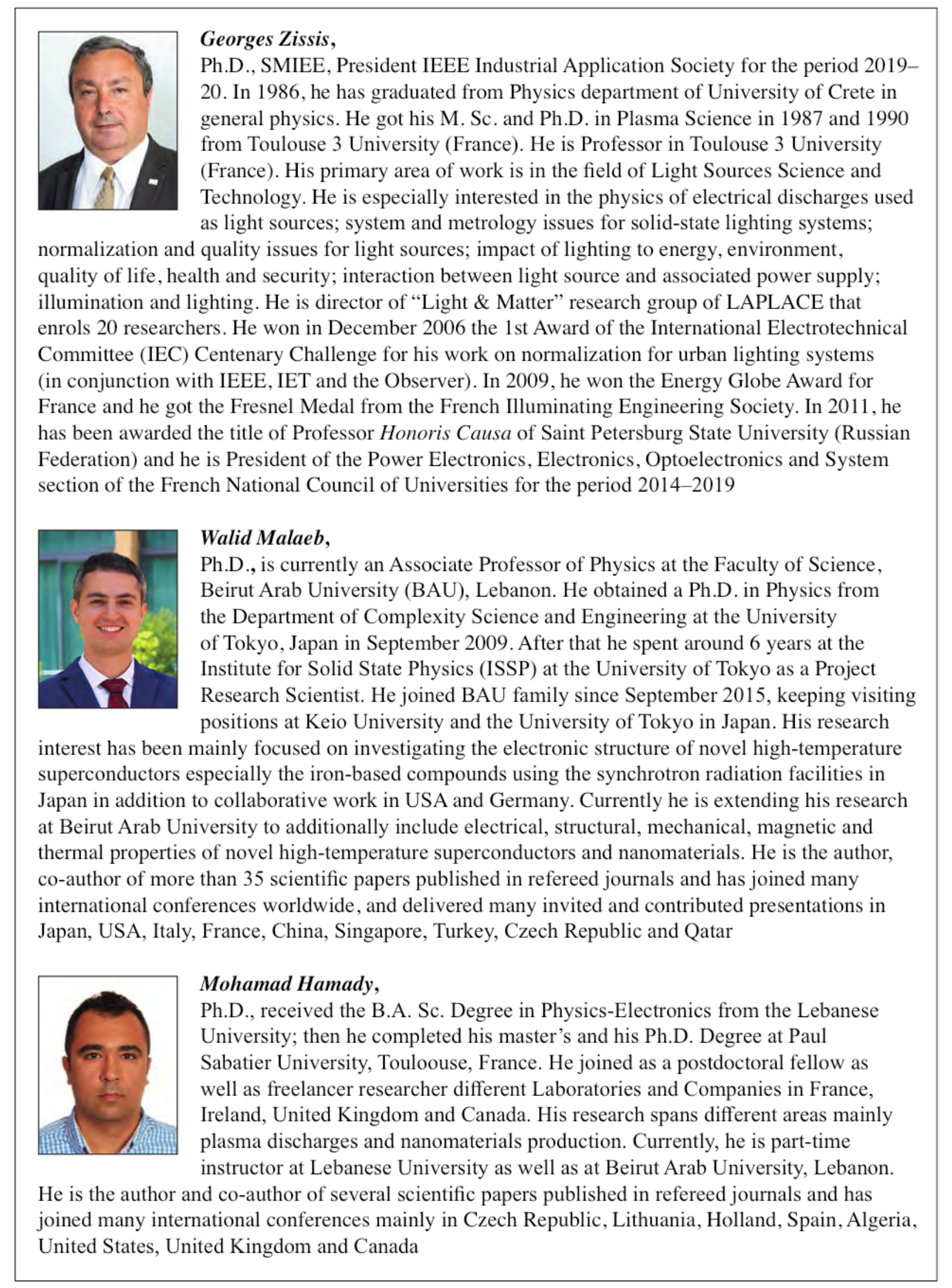

